\title{
Determinación de la dinámica forestal de la matriz de bosques sobre claros vegetales en la Estación Ambiental de Tutunendo, Quibdó, Chocó, Colombia
}

\author{
Determination of forest dynamics of forest on clear matrix plant in Season \\ Environmental Tutunendo, Quibdó, Chocó, Colombia
}

\author{
Juan Mauricio Rincón Gallego', Jovanny Mosquera Pino²
}

\section{Resumen}

Los bosques húmedos del Pacifico colombiano, han sufrido perturbaciones por actividades antropogénicas, como son la minería, la agricultura y el aprovechamiento forestal selectivo de maderas; esta última actividad, crea un hueco vertical sobre los bosques los cuales son llamados claros vegetales, Por esta razón, se realizó el monitoreo de dos claros en la Estación Ambiental de Tutunendo (EAT). La investigación se centra en determinar la relación existente de la matriz y su influencia con la dinámica forestal, identificando especies forestales de interés especial emergentes (brinzales, latizales y fustales) en un periodo de un año (2014) con intervalos de dos meses; por otra parte, se seleccionó un área de $50 \mathrm{~m}$ de radio alrededor de cada claro para identificar los estadios sucesionales de las especies forestales presentes en la matriz del bosque, estado de desarrollo y su posible influencia en la presencia o ausencia de las especies vegetales emergentes en los claros. Para el claro número uno, se identificaron 67 individuos, representados en 12 especies forestales y agrupadas en 8 familias, entre las que se destacan, Arecaceae y Annonaceae. Para el claro número dos, se registraron 38 individuos, representadas en 8 especies pertenecientes a 8 familias, siendo Arecaceae y Bombacoideae, las familias más abundantes. En relación con las especies forestales presentes en la matriz de cada uno de los claros, se pudo observar que las especies forestales que emergen en los claros son influidas directamente en su dispersión y crecimiento por los árboles presentes en la matriz.

Palabras clave: Bosque húmedo, Claros vegetales, Composición florística, Dinámica natural, Matriz de claros de bosques.
Ingeniero Agroforestal, contratista del Instituto de Investigaciones Ambientales del Pacífico (IIAP), Quibdó, Colombia.

e-mail: coltanz07@hotmail.com

2 Ingeniero Agroforestal, Investigador Asociado II del Instituto de Investigaciones Ambientales del Pacífico (IIAP), Coordinador proyecto Monitoreo de Bosques, Quibdó, Colombia.

e-mail: jmosquera@iiiap.org.co

Recibido: 10 de diciembre de 2012 Aceptado: 19 de marzo de 2013

\begin{abstract}
The humid forests of the Colombian Pacific, have suffered disturbances by anthropogenic activities such as mining, agriculture and selective logging of timber; the latter activity, creates a vertical hole on forests which are called light vegetables. For this reason, monitoring of two clear in the Environmental Station was performed Tutunendo (EAT). The investigation is focused on determining the relationship of the parent and its influence forest dynamics, identifying tree species of special interest emerging (seedlings, saplings and fustales) over a period of one year 2014 with time intervals two months. Moreover, an area of $50 \mathrm{~m}$ radius was selected around each course to identify successional stages of forest species present in the matrix of forest, state of development and its possible influence on the presence or absence of emerging plant species clear. To the clear number one, 67
\end{abstract}




\section{Dinámica forestal y bosques sobre claros vegetales. JM Rincón, J Mosquera}

individual represented in 12 forest species and grouped in 8 families, among which stand out Arecaceae, Annonaceae were identified. To the clear number two, 38 individuals, represented in 8 species belonging to 8 families, being Arecaceae and Bombacoideae), the mostabundantfamilies were recorded. Regarding forest species present in the matrix of each of the light, it was observed that forest species that emerge in clearings are influenced directly in their dispersal and tree growth present in the matrix.

Keywords: Floristic composition, Forest clearings matrix, Light vegetables, Natural dynamics, Wet forest.

\section{Introducción}

El bosque húmedo tropical se describe como un mosaico de parches de diferentes tamaños y edades de crecimiento, originando los claros de luz por la caída de árboles (Baur 1968, Whitmore 1989), quienes sostienen que en estos bosques muchas especies de árboles dependen del estado del dosel para una o todas las etapas de su vida, desde el crecimiento en el claro hasta su madurez.

Como consecuencia de la extracción selectiva y caída natural de los árboles en el bosque tropical se forman los claros; en estos claros ingresa mayor cantidad de radiación solar, hecho que induce al establecimiento de la regeneración natural, sobre la que, por lo general existe poca información sobre todo en lo que se refiere a la identidad de las especies que aprovechan esta oportunidad, de tal modo que se ignora si pertenecen a poblaciones lejanas diferentes.

Según Whitmore (1989) con el paso del tiempo se puede observar una regeneración natural muy heterogénea en altura, por tal razón es necesario estratificarla y en ese sentido, se debe dividir el proceso sucesional comprendido en tres fases: fase del claro, fase de identificación y fase madura; además, Vidaurre (1991) dice la regeneración natural del bosque continúa siendo el método más deseable de manejo, que es la sustentabilidad concebida para mantener la producción de la madera y para proteger a la vez la ecología del bosque tropical. Sin embargo, con una frecuencia mucho mayor, en la formación de claros, producto de la acción antrópica, específicamente los producidos por la extracción selectiva de árboles, porque según Benítez (1996) en la dinámica sucesional iniciada, interviene una combinación de factores tanto endógenos como exógenos a la comunidad y además se pueden diferenciar 3 zonas dentro del claro natural producido, como la zona del tocón, zona del tronco y zona de la copa.

Por otra parte Nadia et al. (2011), sostienen que la extracción de maderas de bosques naturales reduce la complejidad estructural de la vegetación. Según Cannon et al. (1994), Ochoa (1998) y Padmawathe et al. (2004), induce a cambios en la adecuación de las comunidades de animales silvestres y Johns (1988), Ochoa (2000), Bin Hussin y Francis (2001), Ghazoul y Hill (2001) Ochoa y Soriano (2001), Plumptre y Grieser (2001), Soriano y Ochoa (2001) indican que genera reducción de las fuentes de agua, fragmentación del hábitat y erosión de los suelos (Laurance et al. 2001).

Este trabajo busca determinar la dinámica forestal de la matriz de bosque sobre claros vegetales en la Estación Ambiental de Tutunendo (EAT), en el departamento del Chocó, para lo cual pretende identificar especies emergentes forestales de interés especial en dos claros, así como aquellas en estado latizal y fustal presentes en dos bosques continuos a los claros y evaluar la influencia de la matriz de bosque sobre los mismos.

\section{Metodología}

Area de estudio. El estudio se realizó en dos sitios ubicados en la EAT, municipio de Quibdó, Chocó, Colombia. La EAT se encuentra localizada en la parte nororiental del municipio de Quibdó, a los $5^{\circ} 45^{\prime}$ latitud norte y $76^{\circ} 30^{\prime}$ longitud oeste (Ramírez y Galeano 2011), con una altura promedio de $120 \mathrm{msnm}$, humedad relativa de $80 \%$, temperatura de $28^{\circ} \mathrm{C}$ y una precipitación de $7800 \mathrm{~mm}$. La zona de estudio se encuentran dentro del grupo cinco, pie de monte y colinas bajas, en la subregión ecogeográfica cinco, central norte del Chocó Biogeográfico (Poveda et al. 2004). De acuerdo con lo indicado por Holdridge (1996), la mayor parte del territorio se encuentra ubicado en la zona de vida de bosque pluvial tropical (bp-T) a bosque muy húmedo tropical (bmh-T). Los habitantes de la zona de estudio son de etnia afrocolombiana y sus principales actividades son el comercio de madera y la agricultura (Figura 1).

Ubicación de los claros vegetales. El claro $\mathrm{N}^{\circ} 1$, se ubica a una distancia de 45 minutos a pie por el camino terciario que conduce de la cabaña principal de la EAT hasta el claro a $5^{\circ} 44^{\prime} 41.7^{\prime \prime}$ latitud norte y $76^{\circ} 31^{\prime} 24.2$ " longitud, es un claro que presenta un área de $50 \mathrm{mx} 50 \mathrm{~m}$ y 10 cuadrantes de $10 \mathrm{mx} 10 \mathrm{~m}$. Además, posee suelos de planicie con algunas pendientes, como se pudo observar en los cuadrante 6 y 7 , no posee zonas inundables; los suelos tiene gran cantidad de hojarasca y arcilla. El claro $\mathrm{N}^{\circ} 2$ se ubica a unos $200 \mathrm{~m}$ más arriba de la entrada principal de la EAT por la carretera que conduce al municipio de Medellín, Antioquia y luego se ingresa a pie unos 20 pasos, se localiza geográficamente a los $05^{\circ} 44^{\prime} 58.2^{\prime \prime}$ norte y $076^{\circ} 31^{\prime} 10.4^{\prime \prime}$ oeste y posee 2 cuadrantes de $10 \mathrm{mx} 10 \mathrm{~m}$ respectivamente, este claro de bosque, como el anterior, no es de zona inundable, se pudo observar, que ha sido sometido a más intervención antrópica que el claro $\mathrm{N}^{\circ} 1$, con características geográficas casi similares y composición florística un tanto diferente (Figura 2). 
Bioetnia Volumen 10, 2013

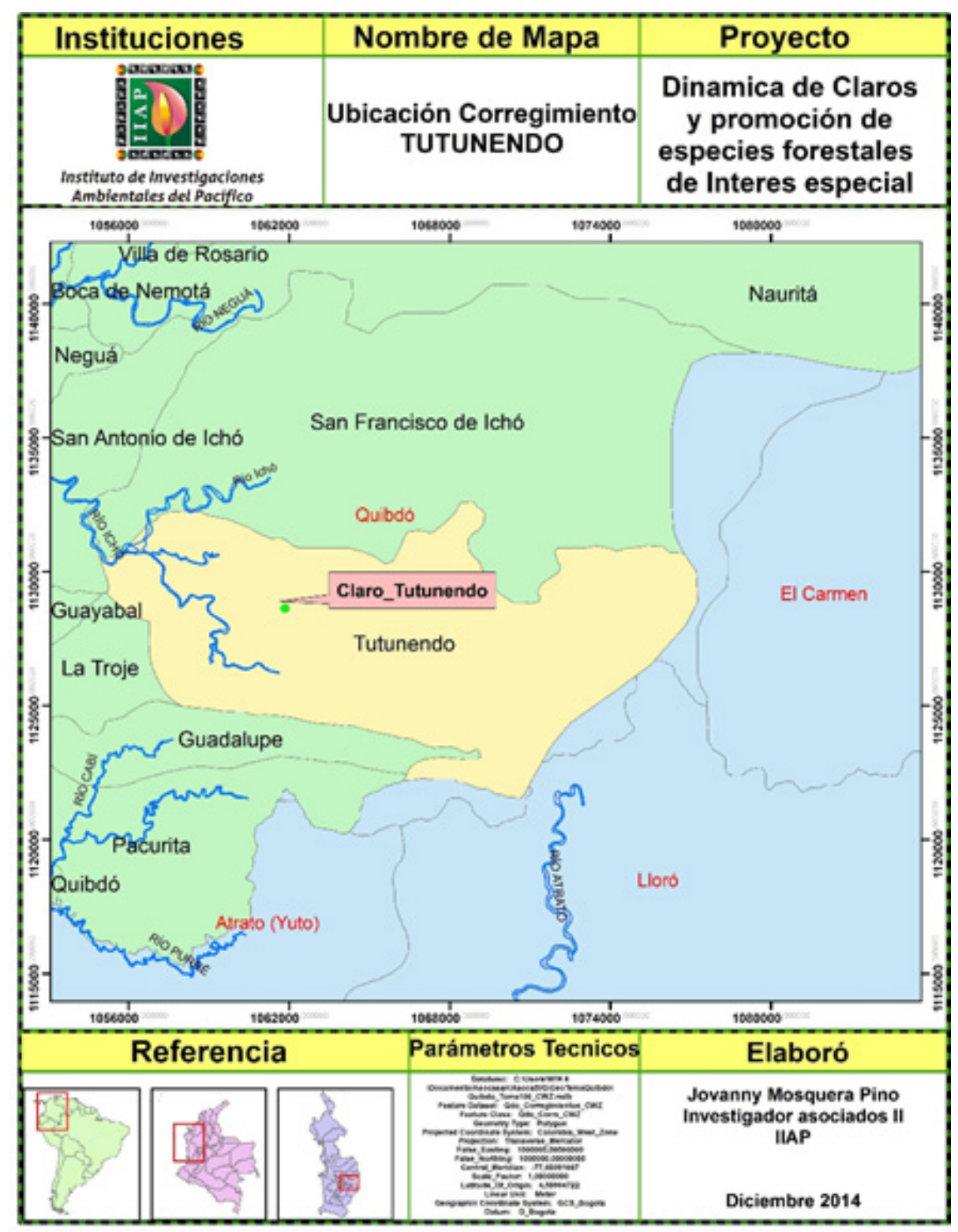

Figura 1. Ubicación área de estudio.

\section{Métodos}

Identificación y monitoreo de especies forestales de interés especial emergentes en los claros. Las áreas de los claros se delimitaron con cinta reflexiva, geoposicionando cada uno de los vértices que formará la superficie del claro y subdividiéndolo en subcuadrantes $10 \mathrm{mx} 10 \mathrm{~m}$, donde se registraron durante 12 meses con intervalos de 60 días, las especies forestales de interés especial (ecológico, comercial, medicinal o comestible) en estadío brinzal, latizal y fustal. Se tomaron testimonio a los investigadores botánicos comunitarios de la zona, para identificar en campo el nombre común de cada individuo y registrarlo en los instrumentos de recolección de información elaborados para este fin, además se etiquetaron y se identificaron utilizando claves taxonómicas. A las especies identificadas se les registró altura total, diámetros y altura comercial para las especies en estadío fustal, asimismo, se registró el estado fitosanitario (Figura 3).

Registro de información en la matriz de bosque asociada con los claros. Se trazó como estrategia metodológica para el levantamiento de la información un barrido de todas las especies que se encontraban dentro de las matrices de los claro de bosque $\mathrm{N}^{\circ} 1$ y $\mathrm{N}^{\circ} 2$, teniendo en cuenta las diferencias en los estadíos sucesionales emergentes en estos, para establecer si las primeras están influyendo en 

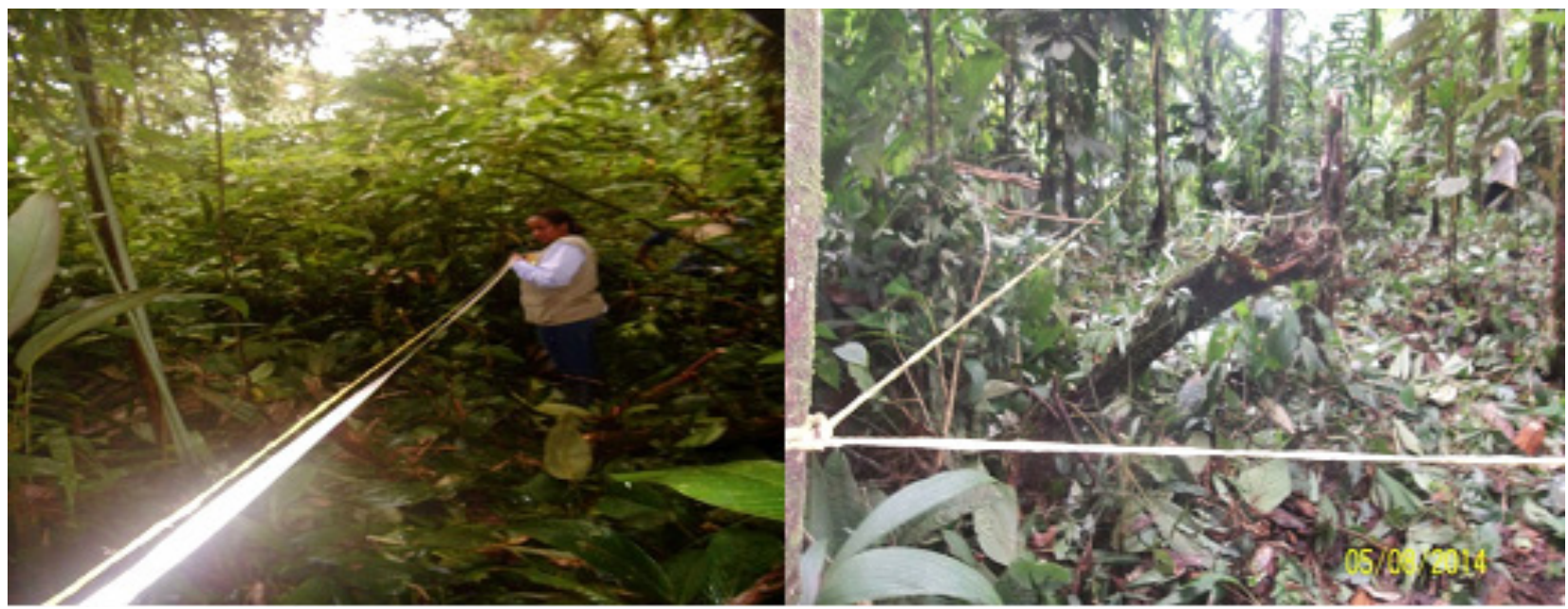

Figura 2. Contorno claros $\mathrm{N}^{\circ} 1$ y $\mathrm{N}^{\circ} 2$.

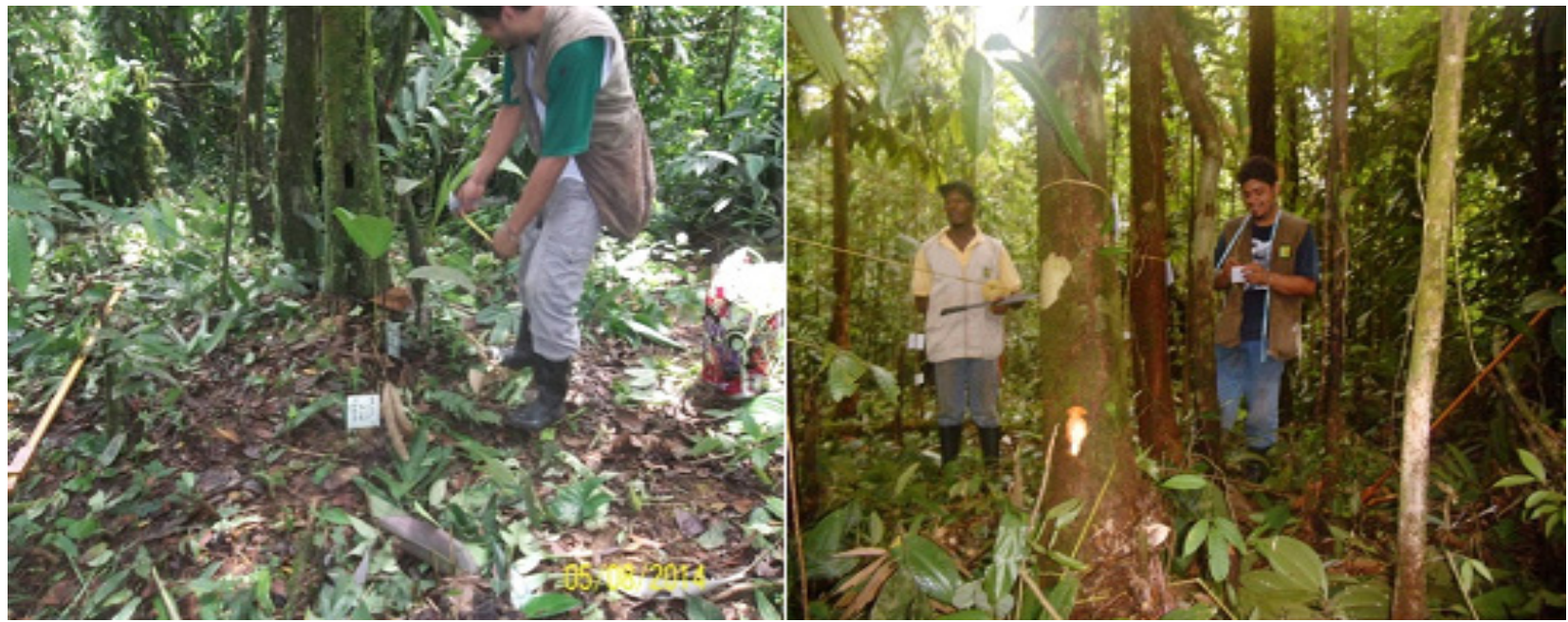

Figura 3. Identificación y etiquetado de individuos.

la dinámica sucesional de los claros, en sisa y de forma circular, de igual manera, se realizó la identificación de las especies vegetales en estadía latizal y fustal siguiendo la metodología descrita y registrando el estado de reproducción (Figura 4).

Análisis y sistematización de la información. La determinación del material y el análisis de la información se realizó siguiendo a Rangel-Ch. (2004) y Mahecha (1997), además de la ayuda de claves taxonómicas, descripciones y las bases de datos como: Herbario Nacional Colombiano (COL), Jardín Botánico de Missouri (MO), New York Botanicals Garden (NY) y Real Jardín Botánico (KEW). De la misma manera, la compilación y tabulación de los datos se realizó mediante la utilización de base de datos en Office Excel tomando como referente los cuadros y fórmulas establecidas en la guía técnica del Minambiente (2002), las cuales permitieron generar tablas y gráficas dinámicas que facilitaron el análisis de la información. En lo relacionado para determinar la composición y estructura de los claros, se realizó exclusivamente tomando en cuenta el tipo de especies en cada sitio, por lo que sus resultados no se miran de manera absoluta, simplemente constituyen una manera de comparar la situación que en materia de escasez se encuentra en cada sitio. Las respectivas variables se calcularon así:

Densidad=Número de individuos/área total del muestreo en hectáreas.

Abundancia relativa $=$ (Número de individuos/Número de individuos en el área muestreada) $* 100$.

Frecuencia $=($ Número de unidades de muestreo/Número total de unidades de muestreo) $* 100$

Frecuencia relativa $=($ Frecuencia absoluta/Sumatoria de las frecuencias absolutas) * 100

Dominancia relativa $=($ Área basal de cada especie $/$ Área basal total )*100

Índice de valor de importancia $(\mathrm{IVI})=$ Abundancia rela- 


\section{Bioetnia Volumen 10, 2013}

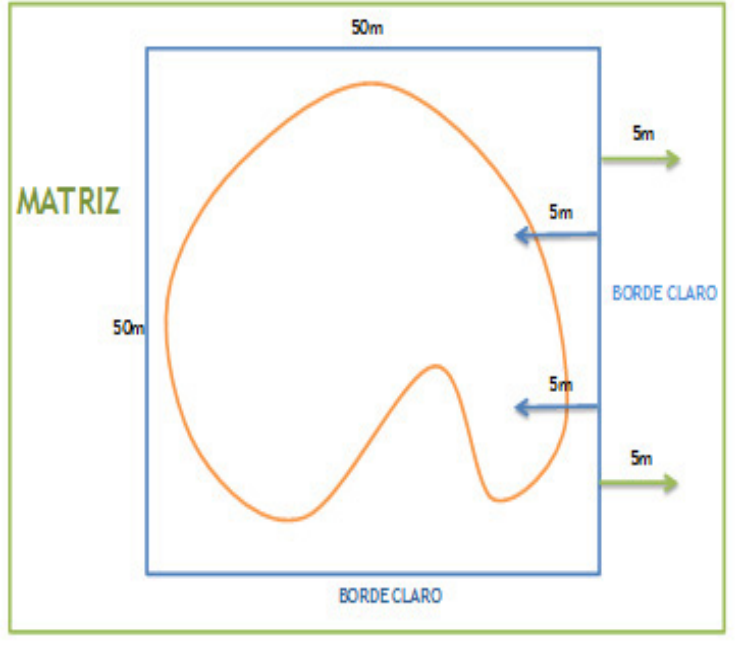

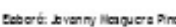

Figura 4. Diseño toma de muestra en el borde y matriz del claro.

tiva $\%+$ Frecuencia relativa $\%+$ Dominancia relativa $\%$.

Por otra parte, el análisis de diversidad, se realizó utilizando el programa estimates versión 9.1.0 determinando la abundancia de cada una de las especies por sitios de muestreos y riqueza específica. También se calculó el índice de valor de importancia (IVI) de las especies y familias para deducir aspectos importantes como la dominancia y especies más representativas

\section{Resultado y discusión}

Composición florística de los claros. En la determinación de la dinámica forestal de la matriz de bosques y su influencia en claros vegetales en la EAT, para el claro $\mathrm{N}^{\circ} 1$ se registraron 67 individuos, pertenecientes a 12 especies y representados por 8 familias (Tabla 1). Las especies más abundantes son: Geonoma cuneata (18) seguida de Pollalata discolor y Cecropia peltata (8 cada uno) y Miconia affinis (7). Por otra parte, en el claro $\mathrm{N}^{\circ} 2$ se registraron 38 individuos, representados en 8 especies y distribuidos en 8 familias, siendo las especies Wetiinia quinaria (12), Ochroma pyramidale (10) y Pollalata discolor (H.B.K.) Arist (8) las más abundantes (Tabla 2).

La Figura 5 muestra que si bien es cierto existe la misma cantidad de familia por especie, esta varía en uniformidad; dicho de otra manera, de las ochos familias registradas en ambos claros, solo tres son frecuentes en los dos sitios, siendo Arecaceae la más abundante con 14 especies en total. Estos datos ratifican la alta riqueza de palmas en Tutunendo (Moreno (2010), donde en sus estudios de maestría dice: "la riqueza de palmas encontrada en las dos localidades estudiadas ( 33 especies en $2.5 \mathrm{ha}$ ), con un promedio de 16 especies en 0.1 ha para Tutunendo y de 13 especies por 0.1 ha para Angostura, está entre las más altas registradas para el neotrópico. El otro dato con una riqueza mayor de palmas es el encontrado por Gentry (1986) para un transecto de un área cercana en Tutunendo, donde registró 17 especies

Tabla 1

Composición florística claro $\mathrm{N}^{\circ} 1$

\begin{tabular}{lllc}
\hline \multirow{2}{*}{ Familia } & \multicolumn{2}{c}{ Nombre } & Abundancia \\
\cline { 2 - 3 } & \multicolumn{1}{c}{ científico } & \multicolumn{1}{c}{ vulgar } & \\
\hline Annonaceae & Guatteriaaff ampliofolia & Cargadero & 1 \\
Arecaceae & Balmeaanomala & Cargadero negro & 1 \\
& Geonomacuneata & Chascarra & 6 \\
& Wetiiniaquinaria & Palma de indio & 3 \\
Asteraceae & Socrateaexorrhiza & Palma meme & 18 \\
Cecropiaceae & Pollalatadiscolor (H.B.K.) Arist & Pliso & 2 \\
Fabaceae & Cecropiapeltata & Yarumo & 8 \\
Melastomataceae & Inga acrocephala & Guamo & 8 \\
& Miconiaaffinis & Hormigo & 4 \\
Moraceae & Castilla elastina Sesse. & Cauchillo & 7 \\
Myristicaceae & Compsoneuraotopa (A.C.Sm.) & Cascajero & 3 \\
Total & & & 6 \\
\hline
\end{tabular}




\section{Dinámica forestal y bosques sobre claros vegetales. JM Rincón, J Mosquera}

de palmas en 0.1 ha", datos que pueden inferir que con la riqueza, frecuencia y abundancia de morfoespecie de interés especial emergentes en ambos claros como Wettinia, estos claros que fueron creados en el año 2007, se encuentran en proceso que permite simular las condiciones iniciales del bosque.

Contrario a lo anterior, Pino (2011) argumenta que en estudios realizados en dos claros de la Estación Ambiental del Alto San Juan, en el municipio de Tadó, la presencia de morfoespecie pertenecientes a la familia Cecropiaceae, son indicadores del grado de perturbación antrópica del sitio y le gremios de heliófilas durables que estas representan.

La Figura 5 ilustra la abundancia de especie presente en el claro $\mathrm{N}^{\circ} 1$, influido por palma meme (Wetiinia quinaria), chascarra (Bactrismaraja), cascajero (Compsoneuraotopa (A.C.Sm.). Además se pudo evidenciar alta presencia brinzales alrededor del claro, las cuales promueven de una $\mathrm{u}$ otra forma a que los claros se regeneren con mayor rapidez; este es otro rasgo que permite emparentar la información registrada con respecto al claro $\mathrm{N}^{\circ} 2$, lo que a su vez también lo hace diferenciar de la matriz del claro $\mathrm{N}^{\circ} 1$, siendo indirectamente proporcional, porque su diversidad de especies botánicas, permitieron determinar que está influido por especies forestales, como el balso (Ochromapyramidale), la palma meme ( $W$. quinaria) esta especie, relaciona el rasgo característico entre los claros de bosque $\mathrm{N}^{\circ} 1$ y $\mathrm{N}^{\circ} 2$, como también el aliso [Pollalatadiscolor (H.B.K.) Arist], seguido por el caraño (Protiumamplum) y sangre gallina (Vismiaglobulifera). Por otra parte, Becerra y Cruz (2007) sostienen que las especies que se encuentra dentro y fuera de los claros de bosque $\mathrm{N}^{\circ} 1$ y $\mathrm{N}^{\circ} 2$, en su gran mayoría presentan un alto valor de uso, bien sea alimenticio, maderable, social, medicinal y ecológico.
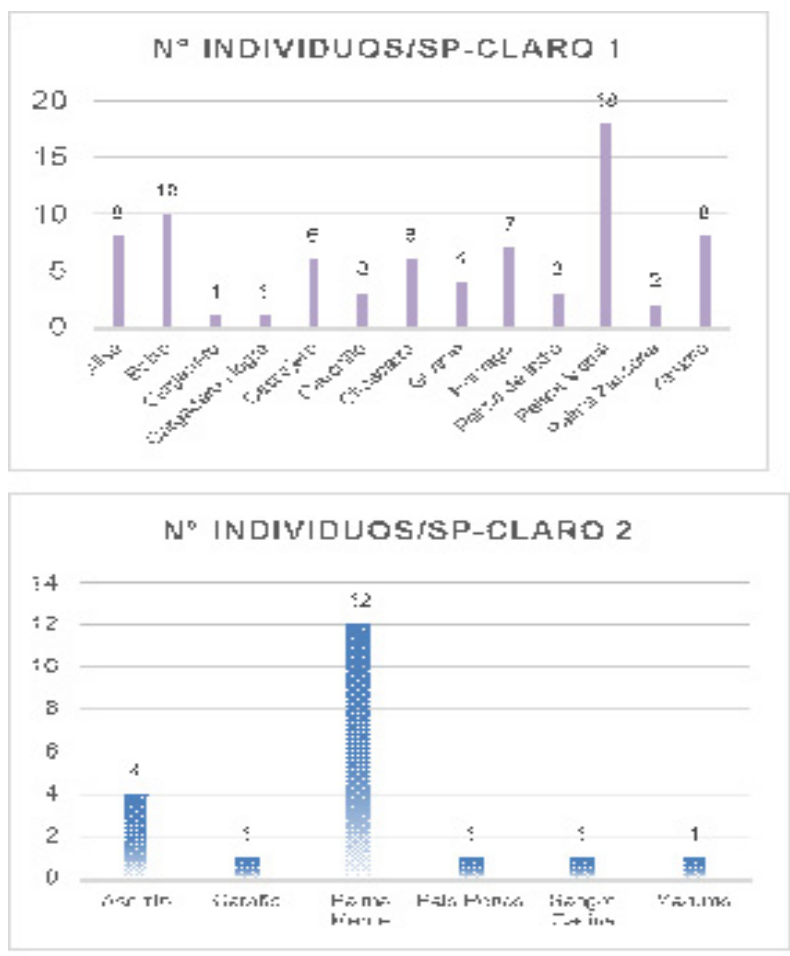

Figura 5. Número de especies en los claros $\mathrm{N}^{\circ} 1$ y $\mathrm{N}^{\circ} 2$.

\section{Conclusiones}

En los claros $\mathrm{N}^{\circ} 1$ y $\mathrm{N}^{\circ} 2$, se determina que la composición florística es influida por tres especies presente en el borde del claro; también se pudo evidenciar que alrededor del claro existe un gran número de especies de habito arbóreo, arbustivo y herbáceo. Por consiguiente, se infiere que en su gran mayoría las especies forestales de interés

Tabla 2

Composición florística claro $\mathrm{N}^{\circ} 2$

\begin{tabular}{lllr}
\hline \multirow{2}{*}{ Familia } & \multicolumn{1}{c}{ Nombre } & \multirow{2}{*}{ Abundancia } \\
\cline { 2 - 3 } & \multicolumn{1}{c}{ científico } & vulgar & \\
\hline Arecaceae & Poliinia quinaria & Plama meme & 12 \\
Asteraceae & Ochromapyramidale & Aliso & 8 \\
Bombacóideas & Protiumamplum & Balso & 10 \\
Burseráceas & Pouruma bicolor Mart. & Caraño & 1 \\
Cecropiaceae & Vismiaglobulifera & Sangre gallina & 1 \\
Clusiaceae & Parkiapendula & Aserrín & 1 \\
Mimosaceae & Simarouba amara & Palo perico & 4 \\
Simaroubaceae & & & 1 \\
Total & & 38 \\
\hline
\end{tabular}




\section{Bioetnia Volumen 10, 2013}

especial presente en los claros emergen por la dispersión de semillas tanto sexual como asexual de estas especies que se encuentran en la matriz del claro.

Todo esto contribuye a la dinámica ecológica de los claros, aportada por la sucesión vegetal, factores climáticos o la fauna asociada con la matriz, lo cual influye a que de una u otra forma las especies de interés especial presente en los claros, se les realice manejo silvicultural para garantizar la supervivencia de las mismas.

Por otra parte, en la matriz del claro del bosque $\mathrm{N}^{\circ}$ 1 , representa una diversidad y abundancia de especies forestales moderadamente leve, por medio del cual se inventariaron 67 individuos, representados en 12 especies forestales, agrupadas en 8 familias, entre las que se destacan Arecaceae, Annonacea, Cecropiaceae, Moraceae y Myristicaceae; con respecto al efecto de borde del claro de bosque $\mathrm{N}^{\circ} 2$, se pudo registrar y connotar que existen características casi similares, entre las especies forestales emergentes en esta matriz del claro de bosque y el borde del claro $\mathrm{N}^{\circ} 1$ y su diferencia básica está en las dimensiones de sus respectivos interlineados trazados, lo que es indirectamente proporcional generando diferencias, pero a su vez, el claro $\mathrm{N}^{\circ} 2$ se emparenta con el claro $\mathrm{N}^{\circ} 1$, al observar la composición florística entre estas dos áreas de matrices de claro de bosque en la EAT.

Por otra parte, se determinó que la matriz de los claros, poseen gran variedad asociada y moderadamente dinámica de especies forestales de interés especial y en algunos casos de uso homeopático o con aplicaciones de medicina tradicional y moderna como en el caso de Simarouba amara que contiene propiedades antimicrobianas.

Además, se pudo evidenciar que existen dinámicas ecológicas pertenecientes a otro gran número de especies arbóreas, arbustos y hiervas alrededor, algunas de estas especies se encuentran en la zona y/o en áreas vecinas, teniendo en cuenta también factores climáticos, relevando el factor luz por brindar al bosque una influencia dinámica de primer rango, debido a que también, es lo que en esencia activa las funciones fotosintéticas de todas las especies botánicas y permiten estimular y promover de una u otra forma que en los claros de bosque, las plantas se regeneren y desarrollen con mayor rapidez en el estadío brinzal, pero que algunas en su gran mayoría, no pasan al estadío de latizal.

\section{Literatura citada}

Baur GN. 1968. The ecological basic of rain forest management. Sydney: Forestrycommission; $499 \mathrm{pp}$.

BenítezME. 1996. Dinámica sucesional en claros producidos por perturbaciones naturales y explotaciones forestales. Rev Forestal Venezol. 40 (2): 21-8.

Becerra TA, Cruz MY.2007. Composición florística y etnobotánica de un bosque pluvial tropical (bp-T) en el corregimiento de Manungara, municipio de Tadó, Chocó, Colombia. [Trabajo de grado]. Quibdó: Facultad de ciencias
Básicas, Universidad Tecnológica del Chocó "Diego Luis Córdoba".

Bonilla N, Cuesta H, Valois H. 2011. Efectos de la extracción forestal sobre la estructura y composición de un bosque pluvial del Pacífico colombiano. Biodivers Neotrop. 1 (1): 48-54

Cannon CH, Peart DR, Leighton M, Kartawinata K. 1994. The structure of lowland rainforest after selective logging in West Kalimantan, Indonesia. Forest Ecol Manag. 67 (1-3): 49-68.

Ghazoul J, Hill J. 2001. The impacts of selective logging on tropical forest invertebrates. En: Fimbel RA, Grajal A, Robinson JG (Eds.). The cutting edge: conserving wildlifein logged tropical forest. New York: Columbia University.

Gentry AH. 1993. Riqueza de especies y composición florística de las comunidades de plantas de la región del Chocó. Caldasia. 15: 71-91.

Holdridge LR. 1996. Ecología basada en zonas de vida. En: Colección Libros y Materiales Educativos $N^{\circ} 83$. San José: IICA. 216 pp.

Johns AD. 1988. Effects of «selective» timber extraction on rain forest structure and composition and some consequences for frugivores and folivores. Biotropica. 20 (1): 31-7.

Laurance WF, Vasconcelos HL, Lovejoy T. 2001. Forest loss and fragmentation in theAmazon, implications for wildlife conservation. Oryx. 34 (1): 39-45.

Mahecha GE. 1997. Fundamentos y metodología para la identificación de plantas. Bogotá: Proyecto BIOPACÍFICO, Ministerio del Medio Ambiente, PNUD-GEF; 282 pp.

Ministerio de Ambiente y Desarrollo Sostenible. 2002. Por la cual se establece el Salvoconducto Nacional para la movilización de productos primarios provenientes de plantaciones forestales, se modifican las resoluciones números 0438 y 1029 de 2001 del Ministerio del Medio Ambiente y se adoptan otras determinaciones. Bogotá: MinAmbiente;14 pp.

Ochoa J. 1998. Análisis preliminar de los efectos del aprovechamiento de maderas sobre la composición y estructura de bosques en Guayana venezolana. Interciencia. 23 (4): 197-205.

Ochoa J. 2000. Efectos de la extracción de maderas sobre la diversidad de mamíferos pequeños en bosques de tierras bajas de la Guayana venezolana. Biotrópica. 32 (1): 146-64

Ochoa J, Soriano PJ. 2001. The effects of logging on nonvolant small mammal communities in neotropical rain forests. En: Fimbel RA, Grajal A, Robinson JG (Eds.). The cuttingedge: conserving wildlife in logged tropical forest. NewYork: Columbia University Press.

Padmawathe R, Qureshi Q, Rawat GS. 2004. Effects of selective logging on vascular epiphyte diversity in a moist lowland forest of Eastern Himalaya, India. Biol Cons. 119 (1): 81-92.

Pino JM. 2011. Dinámica sucesional de claros en la Estación Ambiental del Alto San Juan. Quibdó. Documento sin publicar. 58 pp.

Poveda C, Rojas-P CA, Rudas L1A, Rangel-Ch O. 2004. El Chocó Biogeográfico: Ambiente físico. En: Rangel-Ch JO (ed.). Colombia Diversidad Biótica IV: El Chocó Biogeográfico/Costa Pacifica. Bogotá: Instituto de Ciencias Naturales, Universidad Nacional de Colombia; pp. 1-21.

Plumptre J, Grieser A. 2001. Changes in primate communities following logging disturbance. En: Fimbel RA, Grajal A, Robinson GR (Eds.). The cutting edge: conserving wildlifein logged tropical forest. New York: Columbia University Press.

Ramírez-Moreno G, Galeano G. 2011. Comunidades de palmas en dos bosques del Chocó, Colombia. Caldasia. 33: 315-29.

Rangel-Ch JO (ed.). 2004. Colombia Diversidad Biótica IV: El Chocó Biogeográfico/Costa Pacífica. Bogotá: Instituto de Ciencias Naturales, Universidad Nacional de Colombia.

Soriano PJ, Ochoa J. 2001. The consequences of timber exploitation for bat communities in tropical America. En: Fimbel RA, Grajal A, Robinson JG (Eds.). The cuttingedge: conserving wildlife in logged tropical forest. New York: Columbia University Press.

Vidaurre H. 1991. Regeneración natural. En: Exposición de los avances y resultados del proyecto de estudio en conjunto sobre investigación y regeneración de bosques en la zona amazónica de la República del Perú. Iquitos: Instituto Nacional de Investigación Agraria y Agroindustria (INIAA).

Whitmore TC. 1989. Tropical forest nutrients, where do we stand? A tour the horizon. In: Proctor J (ed.). Mineral nutrients in tropical forest and savanna ecosystems. Oxford: Blackwell Scientific publications; 1-13 pp. 